\title{
REVIEWS
}

\section{Frailty and Intellectual and Developmental Disabilities: a Scoping Review}

\author{
Katherine McKenzie, $\mathrm{MSc}^{1}$, Lynn Martin, $\mathrm{PhD}^{2}$, Hélène Ouellette-Kuntz, $\mathrm{PhD}^{1}$ \\ ${ }^{1}$ Department of Public Health Sciences, Queen's University, Kingston, ON; ${ }^{2}$ Department of Health Sciences, Lakehead \\ University, Thunder Bay, ON, Canada
}

DOI:http://dx.doi.org/10.5770/cgj.19.225

\section{ABSTRACT}

\section{Background}

Individuals with intellectual and developmental disabilities (IDD) are both living longer than in previous generations and experiencing premature aging. Improved understanding of frailty in this aging population may inform community supports and avoid negative outcomes.

\section{Methods}

The objective of this study was to review the literature on frailty and IDD and determine areas for future research and application. The methodological framework for a scoping review as developed by H. Arksey and L. O'Malley was applied to identify and select original studies published since 2000 .

\section{Results}

Seventeen studies were identified; these were based on the work of researchers from four research programs. The studies utilized six measures of frailty, including two frailty indices, the VFQ-ID(-R), the frailty phenotype, and the frailty marker. Frailty was equally studied as an outcome and as predictor for other outcomes (e.g., mobility, falls, care intensity, institutionalization, and survival).

\section{Conclusions}

There is evidence of a growing interest in the measurement of frailty in aging adults with IDD. As in the general population, frailty in this group is associated with many negative outcomes. While a few measures have emerged, more work is required to replicate results, validate tools, and test the feasibility of applying frailty measures in practice and to inform policy.

Key words: frailty, frail, intellectual disabilities, developmental disabilities, epidemiology

\section{INTRODUCTION}

Population aging has become a topic of increasing interest in the last few decades. In 2000, the World Health Organization report brought attention to a particular subset of the aging population: those with intellectual and developmental disabilities (IDD)..$^{(1)}$ It noted that "most adults with intellectual [and developmental] disabilities who live past their third decade are likely to survive into old age ... Numerous adults are surviving into late old age, with some surviving to become centenarians" (p.6). The WHO report also noted that as more people with IDD attain older age, increased needs and further disparities in functional impairment, morbidity, and even mortality can result. The report highlights that special considerations must be given to "the long-term consequences of therapeutic interventions - examples are movement disorders that may result from the prolonged use of neuroleptic medications, and bone demineralization that may occur secondary to the chronic use of certain anticonvulsants." (p.7). Evidently, aging adults with IDD are at risk of additional struggles beyond those normally expected at increased age. In Canada, persons with (IDD) represent between $1-3 \%$ of the population. ( $^{(2)}$ In the province of Ontario, it is projected that the number of seniors $(65+$ years) with IDD will almost double from roughly 6,000 to 10,000 by 2021 . $^{(3)}$

Persons with IDD are higher users of health care, including aging care. In Ontario, adults (18-64 years) with IDD were almost twice as likely to visit the emergency department and to have been hospitalized in the last year as adults without such disabilities. ${ }^{(4)}$ They are also twice as likely to use home care, and are admitted to long-term care approximately 25 years earlier. ${ }^{(5)}$ The World Health Organization report mentioned above urged governments and the academic community to "undertake a course of research that will help further expand knowledge of the ageing process among older adults with intellectual [and developmental] disabilit[ies]" (p.20).(1)

Frailty has become an important construct in relation to aging. In the general population, it has been shown to be a predictor of adverse outcomes, such as institutionalization, falls, and death. ${ }^{(6-8)}$ Despite efforts, a consensus still has not been 
reached on the definition of frailty. ${ }^{(9)}$ Some components of definitions have high agreement: frailty is a clinical syndrome different from a disability and reflects an increase in vulnerability to stressors; ${ }^{(9)}$ it is multidimensional, with cognitive, social, psychological and environmental aspects, in additional to the traditional biological and physical factors; ${ }^{(9,10)}$ and it is correlated with age. ${ }^{(11)}$

Many measures of frailty have been developed over the past couple of decades, ${ }^{(12,13)}$ including the frailty phenotype, ${ }^{(14)}$ the frailty index, ${ }^{(6)}$ the Study of Osteoporotic Fractures (SOF) index, ${ }^{(15)}$ the Fatigue, Resistance, Ambulation, Illness and Loss of weight (FRAIL) index, ${ }^{(16)}$ the Tilburg Frailty Indicator, ${ }^{(17)}$ PRISMA-7, ${ }^{(18)}$ Groningen Frailty Indicator, ${ }^{(19)}$ and the Edmonton Frailty Scale (EFS), ${ }^{(20)}$ among others. The frailty phenotype, developed by Fried et al., ${ }^{(14)}$ describes frailty as the presence of at least three of five key symptoms (weight loss, weakness, poor endurance and exhaustion, low physical activity, and slowness), and pre-frailty as the presence of two of these features. The frailty index, described by Rockwood et al., ${ }^{(6)}$ is a quantitative measure of nonspecific accumulation of deficits, operationalized as the ratio of health deficits present to health deficits measured. The health deficits could be signs, symptoms, diseases, disabilities, or lab measurements.

A systematic review by de Vries et al. ${ }^{(21)}$ described twenty frailty outcome instruments and reported their inclusion of essential frailty factors: nutritional status, physical activity, mobility, strength, energy (physical domain), cognition, mood (psychological domain), and social relations/social support (social domain). While both the frailty index and the frailty phenotype can predict adverse outcomes, ${ }^{(8,22-25)}$ de Vries and colleagues report that only the frailty index can capture all eight frailty factors. Additionally, the frailty index does not have a predefined list of items, which allows for the inclusion of deficits that are appropriate to a specific population. ${ }^{(26,27)}$

It has been recognized that not all measures of health suitable for the general population will produce accurate results among persons with IDD, ${ }^{(28)}$ and frailty is likely one that needs reconsideration in this population. In particular, individuals with IDD have pre-existing and life-long conditions which may incorrectly appear as age-related deficits. These conditions may include reduced mobility, seizure disorders, limited daily functioning, or sensory impairments; ${ }^{(29)}$ these contribute to multi-morbidity experienced by this population. (30) By definition, frailty is the "consequence of age-related decline in many physiological systems", ${ }^{(31)}$ and as a result, a measure of frailty must be positively correlated with age to be valid. ${ }^{(27,32)}$ While pre-existing deficits may contribute to increased vulnerability or increased risk of adverse outcomes, they would not directly contribute to frailty. It is therefore important to re-evaluate both the conceptualization and measurement of frailty to ensure they emphasize change and vulnerability to stressors. ${ }^{(33)}$

After over two decades of researching frailty in the general population, discussions regarding using frailty measures to improve health policy and clinical practice have begun to emerge. ${ }^{(34-38)}$ This is a goal shared by the IDD community; ${ }^{(39,40)}$ however, a better understanding of the current measures used to assess and monitor frailty among community-dwelling adults with IDD is needed. Early identification of frailty in this vulnerable subset of the aging population has the potential to provide an opportunity to put in place needed community supports, which may help to reduce the number of inappropriate admissions to long-term care or institutionalization (re-institutionalization for some).

\section{METHODS}

The aim of this scoping review was to understand frailty and its measurement in IDD, and to determine the next steps of research and implementation. Arksey and O'Malley's ${ }^{(41)}$ methodological framework for developing a scoping review was applied. This framework has five stages: (1) identifying the research question; (2) identifying relevant studies; (3) study selection; (4) charting the data; (5) collating, summarizing and reporting the results.

To identify relevant studies, the following databases were searched for peer-reviewed articles: Medline, PsycINFO, EMBASE, CINAHL, Cochrane Library, EBM Reviews, and Joanna Briggs Institute.

The focus was on articles published between 2000 and December 31st, 2015. This period corresponds to the landmark publication of the WHO review of aging and IDD. ${ }^{(1)}$ Terms related to IDD and frailty were used in all searches, though these sometimes differed slightly between databases. Appendix 1 presents the search strategy for EMBASE, as an example.

Targeted searches of the grey literature (i.e., unpublished material) were also conducted. Here, websites of groups/ agencies concerned with aging/older adults with intellectual and developmental disabilities were reviewed (e.g., Ontario Partnership on Aging and Developmental Disabilities) and key authors were contacted.

To be eligible for review, articles were required to meet the following criteria: be published in English or French, present or discuss a measure of frailty, include adults 50 years of age or older, and present original research. Review articles were excluded.

Studies were first screened, using titles and abstracts, to select the ones that met the eligibility criteria. If full-text articles were available, these were retrieved. All three authors reviewed studies using the inclusion and exclusion criteria. Agreement of at least two members determined whether an article was included.

The full-text articles were read by all three authors. A tracking and review form was used by one of the authors (KM) to collate reviews and identify factors related to frailty, descriptors, themes, and methods used. All three authors discussed and agreed upon common themes and limitations across studies. The information extracted from studies in- 
cluded population characteristics (i.e., study setting, age, sex, level of intellectual disability) frailty measures, purpose of the frailty measurement, key findings, and the authors' suggested next steps.

\section{RESULTS}

\section{Study Selection}

Figure 1 shows the number of studies screened, assessed for eligibility and included in the review. Excluded studies were ones that did not focus on persons with IDD (e.g., mental illness, mild cognitive impairment) $(n=195)$ or on frailty (e.g., specific conditions that may be related to frailty, such as limitations in activities of daily living, oral health issues, medications, infections, sarcopenia) $(n=47)$ per se.

Seventeen $(n=17)$ publications of original research published between 2000 and December 31st, 2015 were retrieved that measured frailty in aging adults with IDD (Table 1). These 17 studies present the work of four research groups from the Netherlands $(n=11),{ }^{(39,42-51)}$ Austria $(n=$ $2),{ }^{(28,33)}$ Canada $(n=3),{ }^{(5,40,52)}$ and Taiwan $(n=1) .{ }^{(53)}$ This includes grey literature (e.g., theses or dissertations), ${ }^{(42,47,52)}$ although some has since been published in peer-reviewed journals. ${ }^{(54,55)}$

\section{Study Setting}

The 11 publications from The Netherlands were from the Healthy Ageing with Intellectual Disability (HA-ID) study;

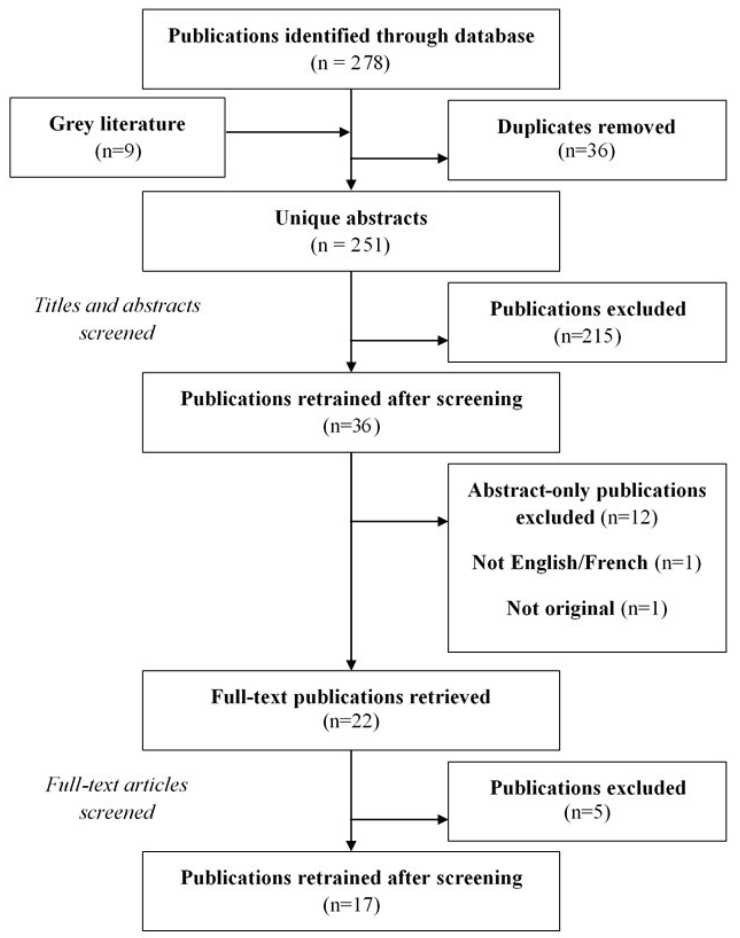

FIGURE 1. Flowchart of search results these analyzed data from a cohort of 1,050 individuals from care providers in The Netherlands providing specialized support (e.g., ambulatory support, day care, residential settings). ${ }^{(39,42-51)}$ The investigators from Austria, as part of the POMONA II project "Health Indicators for People with Intellectual Disabilities", recruited volunteers from eight health areas across the country. ${ }^{(28,33)}$ One Canadian study accessed administratively held health data of individuals with and without IDD in Ontario; ${ }^{(5)}$ the other two Canadian studies accessed data of individuals with IDD who were accessing provincially funded home care in Ontario. ${ }^{(28,33)}$ The study from Taiwan recruited individuals from the voluntary registry, "the Republic of China Foundation for Persons with Down Syndrome".(53)

Age

The HA-ID study included individuals aged 50 years and older. ${ }^{(49,56)}$ Most of the studies using data from the HA-ID cohort reported a mean age of approximately 62 years (SD $=8$ years). ${ }^{(39,42,43,46,47,51)}$ In the articles from the POMONA II project, 190 adults over the age of 18 years were included (mean $=41$ years; range $=18-76$ years) ${ }^{(28,33)}$

The Canadian studies used large, population-level cohorts of adults aged $18-99$ years. ${ }^{(5,40,52)}$ In the largest cohort $(n=$ 51,138 ), approximately half of individuals were between the age of 18 and 44 years. ${ }^{(5)}$ In a cohort of 7,863 individuals assessed for home care, the median age was 57 years old, ${ }^{(40)}$ while in an analysis of a subset of this cohort $(n=3,034)$, the mean age was $54 \pm 17$ years. ${ }^{(52)}$

The study using data from the cross-sectional survey "Healthy Aging Initiatives for Persons with an Intellectual Disability in Taiwan: A Social Ecological Approach (II) People with Down syndrome" analyzed data from 216 individuals over 15 years of age. ${ }^{(53)}$ Only $10 \%$ were over the age of 30 years.

Sex

All studies included both sexes in analyses. Approximately $50-52 \%$ of the HA-ID cohort, ${ }^{(56)}$ and the various cohort subsets, ${ }^{(39,42-51)}$ were men. Similarly, $52 \%$ of the sample of individuals in the Austrian studies were men. ${ }^{(28,33)}$ The studies from Canada represented males and females less equally, with men representing between $48 \%$ and $57 \%$ of the cohorts. $(5,40,52)$ Approximately $62 \%$ of the sample in the studies by Lin et al. ${ }^{(53,57)}$ in Taiwan was male.

\section{Level of IDD}

Studies included wide ranges of levels of IDD from borderline and mild cognitive impairment to severe and profound impairment. In the HA-ID studies, approximately a quarter of the cohort had borderline or mild IDD, while a quarter had severe or profound IDD. ${ }^{(39,42-51)}$ In Brehmer 


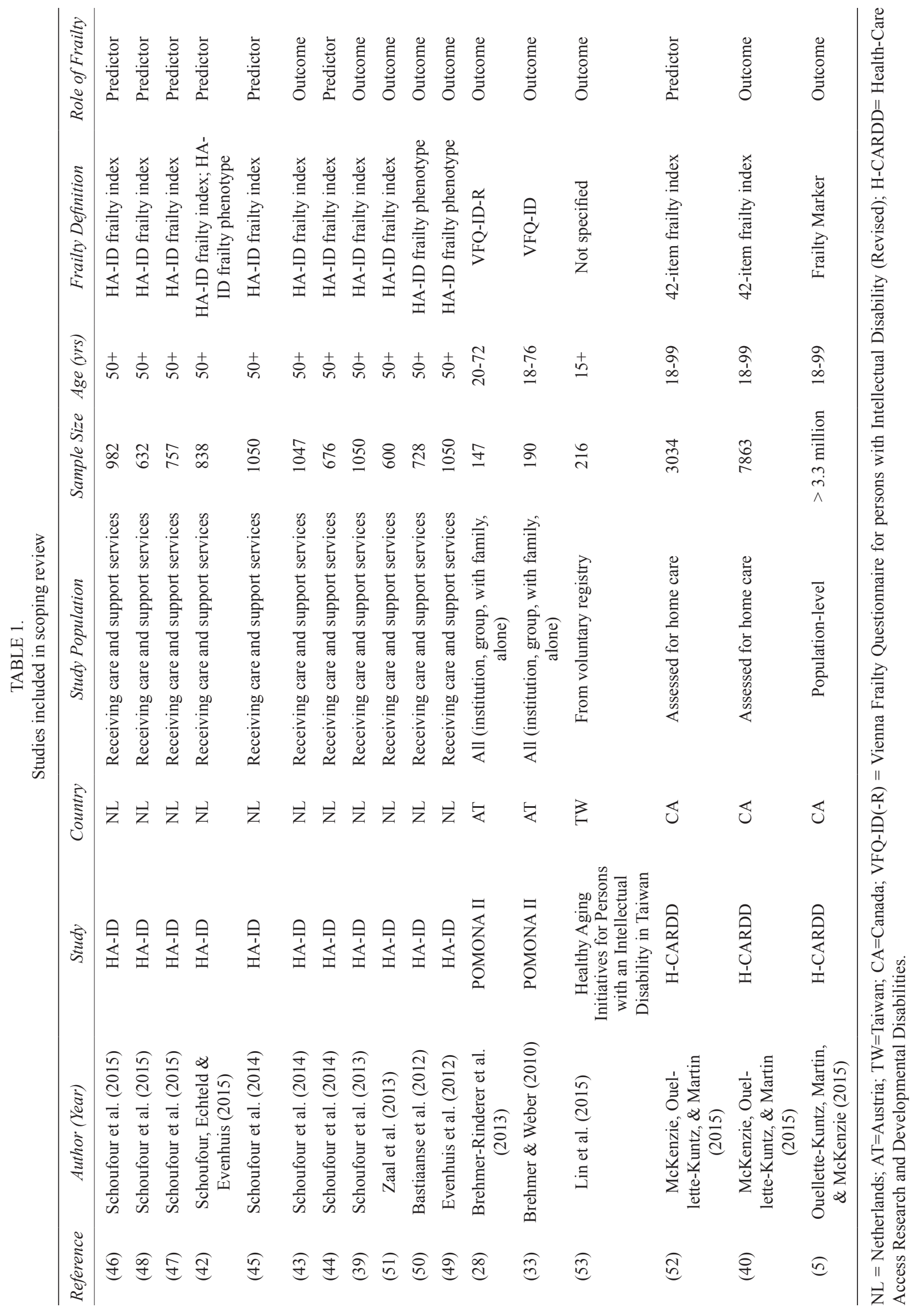


and Weber's Austrian study, ${ }^{(33)} 78 \%$ of individuals could speak for themselves and had low support needs, while the remaining $22 \%$ were non-verbal and had high support needs. In the Canadian studies of home care recipients, approximately half of the cohort had moderate, severe or very severe cognitive impairment. ${ }^{(40,52)}$ The sample from Taiwan included approximately $59 \%$ of individuals with severe or profound IDD. ${ }^{(53,57)}$

Each research program utilized a different method of determining level of IDD. The HA-ID study used scores from psychologists or test assistants, who ascertained levels based on available IQ tests, Vineland scores, and social emotional development. ${ }^{(39)}$ The Canadian studies of home care users used the Cognitive Performance Scale as a proxy for level of IDD ${ }^{(40,52)}$ or provided no information on the level. ${ }^{(5)}$ Lin et al. ${ }^{(53)}$ did not describe how level of IDD was established.

\section{Frailty Measures}

Four distinct measures of frailty were identified. Half of the studies $(n=9)$ utilized the frailty index developed by the HAID study investigators, ${ }^{(39,42-48,51)}$ based on the accumulation of deficits approach. Two studies used the accumulation of deficits approach to develop a frailty index based on data from the Resident Assessment Instrument- Home Care (RAI-HC). $(40,52)$ Three studies applied the frailty phenotype method. $(42,50,58)$ Two studies used the Vienna Frailty Questionnaire for Persons with Intellectual Disabilities (VFQ-ID), ${ }^{(33)}$ and its revision (VFQ-ID-R), ${ }^{(28)}$ which uses a modified accumulation of deficits approach. One study did not specify how frailty had been measured. ${ }^{(53)}$ Lastly, one study measured frailty using a marker derived from the John Hopkins University Adjusted Clinical Group (ACG) System, ${ }^{(5)}$ which classifies a person as frail if he/she has at least one of 81 diagnostic codes. ${ }^{(59)}$

Most studies categorized individuals based on their frailty score. The VFQ-ID-(R) used three levels: frail, pre-frail, and non-frail. Similarly, the frailty phenotype measure also required categorization (frail, pre-frail, and robust/non-frail). The Johns Hopkins frailty marker gives a dichotomous outcome (yes/ no). ${ }^{(5)}$

The frailty index, however, was used differently across studies. In the development of the HA-ID frailty index, ${ }^{(39)}$ and in subsequent analyses, ${ }^{(44,45,47,48,51)}$ it was reported as a continuous variable. Other analyses categorized the frailty index values, including as a dichotomous variable, ${ }^{(43)}$ with three levels, ${ }^{\left({ }^{(2)}\right)}$ with four levels, ${ }^{(51)}$ and with five levels. ${ }^{\left({ }^{(4)}\right)}$ The McKenzie et al. study ${ }^{(40)}$ used categorization to characterize individuals as frail, pre-frail and non-frail.

\section{Key Findings}

\section{Frailty as an Outcome}

Several studies viewed frailty as an outcome of interest, ${ }^{(5,28,33,39,40,43,49-51,53)}$ and reported associations with age, sex, level of intellectual disability, living situation, and other factors.
Age was consistently reported to be associated with frailty, regardless of the method of measuring and categorizing frailty. $(5,28,39,40,43,49)$ Most studies reported that sex was not associated with frailty, $(28,33,39,43,49,53)$ although two studies reported that women had greater odds of frailty, independent of age and other characteristics. ${ }^{(5,40)}$

Most studies evaluated the effect of IDD on frailty. Brehmer and Weber ${ }^{(33)}$ found that those with mild or moderate IDD were more frail, while studies published from the HA-ID cohort consistently reported that adults with severe/ profound IDD were most likely to be frail, ${ }^{(39,43,49)}$ regardless of the measure. Comparing a population of adults with IDD to adults without IDD, the Ouellette-Kuntz et al. study(5) noted that those with IDD were approximately three times more likely to be frail. Without controlling for covariates, the study described a protective effect of cognitive impairment against frailty; however, this was reversed after controlling for age, gender, caregiver status, living situation, and other individual characteristics. ${ }^{(40)}$

There was no agreement with regard to the association between living situation and frailty. In the Austrian study, there was an insignificant increase in risk of frailty in individuals living in institutionalized settings, compared to living alone or with family, while living in an institution or a community-based group home was not significantly associated with increased frailty in the Dutch study. ${ }^{(49)}$ The Canadian study found that compared to living alone, living with family (other than spouse and/or children) or living in a group home, was associated with reduced frailty. ${ }^{(40)}$

Only two studies compared frailty levels in urban versus rural groups, neither of which were significant. ${ }^{(33)}$ Specific diagnoses were also studied in relation to frailty - the presence of dementia, ${ }^{(49)}$ Down syndrome, ${ }^{(39,43,49)}$ mental illness or addiction, ${ }^{(5)}$ and sarcopenia ${ }^{(50)}$ — were positively associated with frailty. Frailty was also associated with relevant or potentially relevant prescription errors. ${ }^{(51)}$

\section{Frailty as a Predictor}

Eight studies used baseline measures of frailty to predict future age-related outcomes, including (instrumental) activities of daily living, ${ }^{(45,53)}$ mobility, ${ }^{(42,45)}$ co-morbidity, ${ }^{(48)}$ falls, ${ }^{(48)}$ fractures, ${ }^{(48)}$ medication use, ${ }^{(48)}$ biochemical markers, ${ }^{(47)}$ care intensity, ${ }^{(44)}$ hospitalizations, ${ }^{(48)}$ admission to long-term care, ${ }^{(52)}$ and survival. ${ }^{(42,46,52)}$

Frailty significantly reduced survival in the community in one four-year follow-up study, ${ }^{(52)}$ and in another study with a three-year follow-up, over $60 \%$ of the frailest individuals died. ${ }^{(46)}$ Frailty was significantly correlated with a decrease in functioning at follow-up, independent of baseline functioning and individual characteristics; ${ }^{(45)}$ and increases in future medication use. ${ }^{(48)}$ Frailty was more closely associated with survival and future functional status than age. ${ }^{(45,46)}$ High levels of frailty were significantly associated with increased care intensity, including institutionalization. ${ }^{(44,52)}$ Frailty was also correlated to some biochemical markers, which 
indicated inflammation, anemia, metabolic issues, and poor renal functioning. ${ }^{(47)}$

Frailty was not predictive of all outcomes examined. After adjusting for sex, age, level of IDD, presence of Down syndrome, and history of outcomes, frailty was not significantly associated with hospitalization, fractures, or falls. ${ }^{(38)}$

\section{Next Steps}

This review also aimed to understand the status of the use of frailty measures in the field of IDD. Currently, the identified measures are used in the context of research, often in stages of development. ${ }^{(28,29,33)}$ As such, the next step is to consider how frailty measures could be applied in practice and policy. This requires a discussion of the limitations in existing studies, and the best strategy for implementing frailty measures in policy and practice. Many of the studies identified in this review suggested future steps.

\section{Study Limitations}

The current body of research on frailty in the field of IDD is limited. A few are prospective cohort studies that face the issue of drop-out and loss-to-follow-up. These longitudinal studies do not repeatedly collect frailty measurements, ${ }^{(44,45,46,52)}$ and so the extent to which frailty status changes over time is unknown. Brehmer and Weber ${ }^{(33)}$ noted the importance of and need for longitudinal research to understand how frailty develops over time.

Samples with overrepresentations of individuals living in care settings or receiving care, rather than in the community, could bias estimates of frailty and increases the likelihood of uncontrolled confounding. Both the HA-ID and the home care studies relied on samples identified through care centres and databases, potentially limiting the generalizability of results to those with higher medical needs. Within the subset of persons with IDD who have higher levels of disability or medical care needs, it may be difficult to obtain some measures needed for the assessment of frailty - for example, walking or answering questions. ${ }^{(49)}$ Additional work is needed to identify appropriate and reliable methods of measuring frailty among those with higher levels of disability.

\section{Implementation}

Some authors view the use of a frailty measure as a method of better describing and identifying individuals with IDD experiencing aging. ${ }^{(42,48)}$ However, it is acknowledged that validation studies must be completed prior to implementation into practice. While the HA-ID frailty index has good predictive validity (e.g., mortality, higher care intensity), ${ }^{(44,46)}$ the results have not been repeated with a different population or with a shorter version better suited for clinical situations. If cut-off scores are used, as they likely would be clinically, these scores should be validated as well. ${ }^{(44,46)}$

To validate a measure of frailty, however, the purpose of the measure must be known. For example, individuals receiving care may be assessed for frailty to either prevent future decline or to address current issues causing frailty. (42) Some have proposed that the most independent, and least-frail, may benefit the most from interventions. ${ }^{(43)}$ Frailty, they proposed, should be stopped in the relatively fit subgroup of the aging population, to alleviate the burden of early frailty. ${ }^{(43,44,53)}$ Doing so would promote healthy aging and functioning, and may provide the most observable benefit. ${ }^{(45)}$ This hypothesis is based on the theory that frailty may be reversible only in its early stages. ${ }^{(9,60)}$

No studies in this review identified a known intervention for the population of adults with IDD, which would be necessary to justify a screening program. ${ }^{(61)}$

Others have suggested frailty could be used to evaluate interventions, or monitor its progression. ${ }^{(46,48)}$

\section{DISCUSSION}

Advocates for the improvement of the health status of older adults with IDD have repeatedly acknowledged the uniqueness of the aging process in this population, ${ }^{(62-66)}$ and expressed the need for a better understanding of aging with IDD. Traditionally, shorter life expectancies, ${ }^{(67-69)}$ early diagnosis of dementia, ${ }^{(57,69,70)}$ and a higher prevalence of various co-morbidities $^{(71-74)}$ have been cited as evidence of premature aging. A report prepared in 2001 by the collaboration of the Aging Special Interest Research Group of the International Association for the Scientific Study of Intellectual Disabilities (IASSID) and the World Health Organization listed the development and evaluation of interdisciplinary interventions for complicated conditions as a high research priority. ${ }^{(75)}$ Over a decade later, the development of frailty measures for this population has occurred; though the literature review spanned the last 15 years, all articles retrieved were published in the last 5 years.

The emerging research focused on IDD and frailty highlights how health indicators focused on physical conditions do not appropriately reflect the age-related risk of vulnerability in this population. ${ }^{(63,70)}$ Individuals with IDD often have lifelong conditions and it is the deterioration, not the presence, of these conditions that indicate worsening well-being. ${ }^{(49,56)}$

In the literature on the general aging population, upwards of 20 unique measures of frailty exist; ${ }^{(13,21)}$ however, variations of two instruments (i.e., the frailty phenotype and the frailty index) are most frequently found in the literature. Given the desire for a validated and multi-item measure applicable to adults with IDD, both the frailty phenotype of Fried et al. ${ }^{(14)}$ and the "accumulation of deficit" frailty indices of Rockwood et al. ${ }^{(6)}$ have been studied in this population. The HA-ID study used both approaches, and reported a preference for the frailty index. The frailty index has the flexibility to include items of all domains of health.

In adults with IDD, frailty has been studied in relation to age-related outcomes, including falls, fractures, health care utilization, functional status, and mortality. It is important to note that these outcomes have largely not been repeated across 
studies, and are the results of only three research studies. The dearth of research in this area is a limitation of this review. In addition, we also note that the authors (KM, LM, HOK) wrote three of the seventeen articles.

While their samples had broad ranges of age and level of IDD, consideration should be given to other factors that could limit generalizability, such as the type of care individuals are receiving (e.g., home care, group home, institutions) and jurisdictional policies towards aging care. In addition, this scoping review identified discrepancies between the risk of frailty based on sex, level of IDD, and living situation; however, many analyses did not adjust for relevant factors, including age. The threats to both internal and external validity present in the identified studies suggest that further research should be conducted. The current studies must be repeated in other populations to assess the replicability of results. This could include in other jurisdictions, care settings, and age groups. Future studies of frailty in this population should also seek to further determine relevant determinants of frailty, using methodology to adjust for covariates.

Many authors proposed validation studies as appropriate future steps; however, consideration should be given to the purpose of measuring frailty before further work is conducted. A measure to inform policy decisions may be vastly different than a measure applied in clinical practice. Within the clinical scope, a frailty screening tool will demand different characteristics than will a diagnostic tool. This includes appropriate levels of sensitivity and specificity, and predictions of outcomes of interest.

If there is a desire to use a frailty measure beyond describing a population's characteristics, consideration of the feasibility of preventing, modifying, or treating frailty, as well as the potential harms of a frailty designation, is warranted. Before a measure of frailty is implemented into clinical practice, research should determine the degree to which frailty indicators add to individual-level prediction of the onset of disability, or other meaningful outcomes, beyond readily available indicators, including age, sex, and co-morbidities. ${ }^{(36)}$ In the general population, reviews ${ }^{(76,77)}$ and ongoing randomized control trials ${ }^{(78,79)}$ have been published that explore possible methods to reduce frailty (e.g., rehabilitation, exercise). Interventions to address frailty in adults with IDD have not been widely explored, although the need to focus on improving quality of life of older adults with IDD has been previously raised. $(75,80)$ The potential for unintended consequences of labelling someone as frail should also be considered.

\section{CONCLUSION}

The past five years have indicated a new interest in the measurement of frailty in aging adults with IDD. Similar to the general population, frailty in the population of adults with IDD is significantly associated with many age-related outcomes. While a few measures have emerged, more work is required to replicate results, validate tools, and test the feasibility of applying frailty measures in settings beyond research studies.

\section{ACKNOWLEDGEMENTS}

This research is funded by Canadian Frailty Network (known previously as Technology Evaluation in the Elderly Network, TVN), supported by Government of Canada through Networks of Centres of Excellence (NCE) Program.

\section{CONFLICT OF INTEREST DISCLOSURES}

The authors declare that no conflicts of interest exist.

\section{REFERENCES}

1. World Health Organization. Ageing and intellectual disabilitiesimproving longevity and promoting healthy ageing: summative report. Geneva, Switzerland: WHO; 2000.

2. Ouellette-Kuntz H, Garcin N, Lewis S, et al. Addressing health disparities through promoting equity for individuals with intellectual disability. Can J Public Health. 2005;96(S2):S8-S22.

3. Ouellette-Kuntz H, Martin L. Applied Health Research Question Report: Aging profiles of adults with and without developmental disabilities in Ontario. Toronto, ON: H-CARDD; 2014.

4. Issacs BJ, Jaakkimainen RL, Barnsley J, et al. Health services utilization. In: Lunsky Y, Klein-Geltink JE, Yates EA, editors. Atlas on the primary care of adults with developmental disabilities in Ontario. Toronto, ON: Institute for Clinical Evaluative Sciences and Centre for Addiction and Mental Health; 2013. p.41-62.

5. Ouellette-Kuntz H, Martin L, McKenzie K. Chapter 6 - A review of health surveillance in older adults with intellectual and developmental disabilities. Int Rev Res Develop Disabil. 2015;48: 151-94.

6. Rockwood K, Mitnitski A, Song X, et al. Long-term risks of death and institutionalization of elderly people in relation to deficit accumulation at age 70. J Am Geriatr Soc. 2006;54(6):975-79.

7. Ensrud KE, Ewing SK, Taylor BC, et al. Frailty and risk of falls, fracture, and mortality in older women: the Study of Osteoporotic Fractures. J Gerontol A Biol Sci Med Sci. 2007;62(7):744-51.

8. Dent E, Chapman I, Howell S, et al. Frailty and functional decline indices predict poor outcomes in hospitalised older people. Age Ageing. 2014;43(4):477-84

9. Rodríguez-Manas L, Féart C, Mann G, et al. Searching for an operational definition of frailty: A Delphi method based consensus statement. The Frailty Operative Definition-Consensus Conference Project. J Gerontol A Biol Sci Med Sci. 2013;68(1):62-67.

10. Woo J, Goggins W, Sham A, et al. Social determinants of frailty. Gerontology. 2005;51(6):402-08.

11. Rockwood K, Song X, MacKnight C, et al. A global clinical measure of fitness and frailty in elderly people. CMAJ. 2005;173(5):489-95.

12. Dent E, Kowal P, Hoogendijk EO. Frailty measurement in research and clinical practice: a review. Eur J Intern Med. 2016;31(1):3-10.

13. Bouillon K, Kivimaki M, Hamer M, et al. Measures of frailty in population-based studies: an overview. BMC Geriatr. 2013;13:64. 
14. Fried LP, Tangen CM, Walston J, et al. Frailty in older adults: evidence for a phenotype. J Gerontol A Biol Sci Med Sci. 2001;56(3):M146-56.

15. Kiely DK, Cupples LA, Lipsitz LA. Validation and comparison of two frailty indexes: The MOBILIZE Boston Study. $J$ Am Geriatr Soc. 2009;57(9):1532-39.

16. Hyde Z, Flicker L, Almeida OP, et al. Low free testosterone predicts frailty in older men: the health in men study. $J$ Clin Endocrinol Metab. 2010;95(7):3165-72.

17. Gobbens RJ, van Assen MA, Luijkx KG, et al. The Tilburg frailty indicator: psychometric properties. JAMA. 2010;11(5):344-55.

18. Raîche M, Hébert R, Dubois MF. PRISMA-7: a case-finding tool to identify older adults with moderate to severe disabilities. Arch Gerontol Geriatr. 2008;47(1):9-18.

19. Schuurmans H, Steverink N, Lindenberg S, et al. Old or frail: what tells us more? J Gerontol A Biol Sci Med Sci. 2004;59(9):M962-65.

20. Rolfson DB, Majumdar SR, Tsuyuki RT, et al. Validity and reliability of the Edmonton Frail Scale. Age Ageing. 2006;35(5):526-29.

21. de Vries NM, Staal JB, van Ravensberg CD, et al. Outcome instruments to measure frailty: a systematic review. Ageing Res Rev. 2011;10(1):104-14.

22. Drubbel I, Numans ME, Kranenburg G, et al. Screening for frailty in primary care: a systematic review of the psychometric properties of the frailty index in community-dwelling older people. BMC Geriatr. 2014;14(1):27.

23. Hogan DB, Freiheit EA, Strain LA, et al. Comparing frailty measures in their ability to predict adverse outcome among older residents of assisted living. BMC Geriatr. 2012;12:56.

24. Blodgett J, Theou O, Kirkland S, et al. Frailty in NHANES: comparing the frailty index and phenotype. Arch Gerontol Geriatr. 2015;60(3):464-70.

25. Ravindrarajah R, Lee DM, Pye SR, et al. The ability of three different models of frailty to predict all-cause mortality: results from the European Male Aging Study (EMAS). Arch Gerontol Geriatr. 2013;57(3):360-68.

26. Theou O, Rockwood K. Should frailty status always be considered when treating the elderly patient? Aging Health. 2012;8(3):261-71.

27. Schoufour JD, Evenhuis HM, Mitnitski A, et al. The Benefits of a frailty index for people with intellectual disability: a commentary. J Policy Pract Intellect Disabil. 2015;12(3):232-34.

28. Brehmer-Rinderer B, Zeilinger EL, Radaljevic A, et al. The Vienna Frailty Questionnaire for Persons with Intellectual Disabilities—revised. Res Dev Disabil. 2013;34(6):1958-65.

29. van Schrojenstein Lantman-de Kalk HM, Walsh PN. Managing health problems in people with intellectual disabilities. BMJ. 2008;337:a2507.

30. Cooper SA, McLean G, Guthrie B, et al. Multiple physical and mental health comorbidity in adults with intellectual disabilities: population-based cross-sectional analysis. BMC Fam Pract. 2015;16:110. p.752.

31. Clegg A, Young J, Iliffe S, et al. Frailty in elderly people. The Lancet. 2013;381(9868):752-62.
32. Rockwood K, Hogan DB, MacKnight C. Conceptualisation and measurement of frailty in elderly people. Drugs Aging. 2000;17(4):295-302.

33. Brehmer B, Weber G. Frailty vs. disability distinctions in people with intellectual disabilities. J Policy Pract Intellect Disabil. 2010;7(1):49-58.

34. Lacas A, Rockwood K. Frailty in primary care: a review of its conceptualization and implications for practice. BMC Med. 2012;10:4.

35. Lepeleire J De, Iliffe S, Mann E, et al. Frailty: an emerging concept for general practice. Br J Gen Pract. 2009;59(562):e177-82.

36. Sourial N, Bergman H, Karunananthan S, et al. Implementing frailty into clinical practice: a cautionary tale. J Gerontol A Biol Sci Med Sci. 2013;68:12.

37. Martin FC, Brighton P. Frailty: different tools for different purposes? Age Ageing. 2008;37(2):129-31.

38. Inouye SK, Studenski AS, Tinetti ME, et al. Geriatric syndromes: clinical, research, and policy implications of a core geriatric concept. J Am Geriatr Soc. 2007;55(5):780-91.

39. Schoufour JD, Mitnitski AB, Rockwood K, et al. Development of a frailty index for older people with intellectual disabilities: results from the HA-ID study. Res Dev Disabil. 2013;34(5):1541-55.

40. McKenzie K, Ouellette-Kuntz H, Martin L. Using an accumulation of deficits approach to measure frailty in a population of home care users with intellectual and developmental disabilities: a descriptive study. BMC Geriatr. 2015;15:170.

41. Arksey H, O'Malley L. Scoping studies: towards a methodological framework. Int J Soc Res Methodol. 2005;8(1):19-32.

42. Schoufour JD, Echteld MA, Evenhuis HM. Comparing two frailty measures in their ability to predict mortality among older people with intellectual disabilities (Chapter 10). In: Schoufour JD, editor. Frailty in people with intellectual disabilities: frequency, determinants and consequences. Rotterdam, The Netherlands: Erasmus University; 2015. p.165-78.

43. Schoufour JD, van Wijngaarden J, Mitnitski A, et al. Characteristics of the least frail adults with intellectual disabilities: a positive biology perspective. Res Dev Disabil. 2014;35(1):127-36.

44. Schoufour JD, Evenhuis HM, Echteld MA. The impact of frailty on care intensity in older people with intellectual disabilities. Res Dev Disabil. 2014;35(12):3455-61.

45. Schoufour JD, Mitnitski A, Rockwood K, et al. Predicting disabilities in daily functioning in older people with intellectual disabilities using a frailty index. Res Dev Disabil. 2014;35(10):2267-77.

46. Schoufour JD, Mitnitski AB, Rockwood K, et al. Predicting 3 -year survival in older people with intellectual disabilities using a frailty index. J Am Geriatr Soc. 2015;63(3):531-36.

47. Schoufour JD, Echteld MA, Boonstra A, et al. Biochemical measures and frailty in people with intellectual disabilities. In: Schoufour JD, editor. Frailty in people with intellectual disabilities: frequency, determinants and consequences. Rotterdam: Erasmus Universiteit Rotterdam; 2015. p.77-90.

48. Schoufour JD, Echteld MA, Bastiaanse LP, et al. The use of a frailty index to predict adverse health outcomes (falls, fractures, 
hospitalization, medication use, comorbid conditions) in people with intellectual disabilities. Res Dev Disabil. 2015;38:39-47.

49. Evenhuis HM, Hermans H, Hilgenkamp TIM, et al. Frailty and disability in older adults with intellectual disabilities: results from the Healthy Ageing and Intellectual Disability study. $J$ Am Geriatr Soc. 2012;60(5):934-38.

50. Bastiaanse LP, Hilgenkamp TIM, Echteld MA, et al. Prevalence and associated factors of sarcopenia in older adults with intellectual disabilities. Res Dev Disabil. 2012;33(6):2004-12.

51. Zaal RJ, van der Kaaij ADM, Evenhuis HM, et al. Prescription errors in older individuals with an intellectual disability: prevalence and risk factors in the Healthy Ageing and Intellectual Disability Study. Res Dev Disabil. 2013;34(5):1656-62.

52. McKenzie K, Ouellette-Kuntz H, Martin L. Frailty as a predictor of institutionalization among adults with intellectual and developmental disabilities using a frailty index and the interRAI CHESS Scale. In: McKenzie K, editor. Aging with intellectual and developmental disabilities: the effect of frailty and health instability in home care users on admission to long-term care. Kingston, ON: Queen's University; 2015. p.70-99.

53. Lin JD, Lin LP, Hsu SW, et al. Are early onset aging conditions correlated to daily activity functions in youth and adults with Down syndrome? Res Dev Disabil. 2015;36(161):532-36.

54. Schoufour JD, Echteld MA, Boonstra A, et al. Biochemical measures and frailty in people with intellectual disabilities. Age Ageing. 2016;45(1):142-48.

55. McKenzie K, Ouellette-Kuntz H, Martin L. Frailty as a predictor of institutionalization among adults with intellectual and developmental disabilities. Intellect Dev Disabil. 2016;54(2):123-35

56. Hilgenkamp TIM, Bastiaanse LP, Hermans H, et al. Study healthy ageing and intellectual disabilities: recruitment and design. Res Dev Disabil. 2011;32(3):1097-106.

57. Lin JD, Chen WX, Hsu SW, et al. Primary caregivers' awareness and perception of early-onset dementia conditions in adolescents and young and middle-aged adults with Down syndrome. Res Dev Disabil Disabil. 2014;35(9):1934-40.

58. Evenhuis HM, Schoufour J, Echteld M. Frailty and intellectual disability: a different operationalization? Dev Disabil Res Rev. 2013;18(1):17-21.

59. Bronskill S, Carter MW, Costa AP, et al. Aging in Ontario :an ICES Chartbook of health service use by older adults [Internet]. Sciences-New York. 2010. Available from: http://www.ices. on.ca/Publications/Atlases-and-Reports/2010/Aging-in-Ontario

60. Martone AM, Onder G, Vetrano DL, et al. Anorexia of aging: a modifiable risk factor for frailty. Nutrients. 2013;5(10):4126-33.

61. Wilson J, Jungner G. Principles and practice of screening for disease. Arch Intern Med. 1969;123(3):349.

62. Fisher K, Kettl P. Aging with mental retardation. Geriatrics. 2005;60(4):26-29.

63. Lifshitz H, Merrick J. Aging among persons with intellectual disability in Israel in relation to type of residence, age, and etiology. Res Dev Disabil. 2004;25(2):193-205.
64. Carmeli E. We need to empower health prevention of aged people with intellectual disability [editorial]. J Aging Sci. 2013;1(3):1-2.

65. Kim NH, Hoyek GE, Chau D. Long-term care of the aging population with intellectual and developmental disabilities. Clin Geriatr Med. 2011;27(2):291-300.

66. McCarron M, Lawlor BA. Responding to the challenges of ageing and dementia in intellectual disability in Ireland. Aging Ment Health. 2003;7(6):413-17.

67. Coppus AMW. People with intellectual disability: what do we know about adulthood and life expectancy? Dev Disabil Res Rev. 2013;18(1):6-16.

68. Patja K, Iivanainen $\mathrm{M}$, Vesala $\mathrm{H}$, et al. Life expectancy of people with intellectual disability: a 35-year follow-up study. J Intellect Disabil Res. 2000;44(5):591-99.

69. Coppus A, Evenhuis HM, Verberne GJ, et al. Dementia and mortality in persons with Down's syndrome. J Intellect Disabil Res. 2006;50(10):768-77.

70. Patti P, Amble K, Flory M. Placement, relocation and end of life issues in aging adults with and without Down's syndrome: a retrospective study. J Intellect Disabil Res. 2010;54(6):538-46.

71. Janicki MP, Dalton AJ, Henderson CM, et al. Mortality and morbidity among older adults with intellectual disability: health services considerations. Disabil Rehabil. 1999;21(5-6):284-94.

72. Dykens EM. Aging in rare intellectual disability syndromes. Dev Disabil Res Rev. 2013;18(1):75-83.

73. Bigby C, Webber R, Bowers B, et al. A survey of people with intellectual disabilities living in residential aged care facilities in Victoria. J Intellect Disabil Res. 2008;52(5):404-14.

74. Haveman MJ. Disease epidemiology and aging people with intellectual disabilities [Internet]. $J$ Policy Pract Intellect Disabil. 2004;1(1):16-23. Available from: http://doi.wiley. com/10.1111/j.1741-1130.2004.04003.x

75. Evenhuis $\mathrm{H}$, Henderson $\mathrm{CM}$, Beange $\mathrm{H}$, et al. Healthy ageing - adults with intellectual disabilities: physical health issues. J Appl Res Intellect Disabil. 2001;14(3):175-94.

76. Theou O, Stathokostas L, Roland KP, et al. The effectiveness of exercise interventions for the management of frailty: a systematic review. J Aging Res. 2011;2011:569194.

77. Kelaiditi E, van Kan GA, Cesari M. Frailty: role of nutrition and exercise. Curr Opin Clin Nutr Metab Care. 2014;17(1):32-39.

78. Fairhall N, Aggar C, Kurrle SE, et al. Frailty Intervention Trial (FIT). BMC Geriatr. 2008;8(1):27.

79. Cameron ID, Fairhall N, Gill L, et al. Developing interventions for frailty. Adv Geriatr. 2015;2015:845356.

80. Carmeli E, Imam B. Health promotion and disease prevention strategies in older adults with intellectual and developmental disabilities. Front Public Health. 2014;2:31

Correspondence to: Hélène Ouellette-Kuntz, PhD, Department of Public Health Sciences, Queen's University \& Ongwanada, 191 Portsmouth Avenue, Kingston, ON K7M 8A6, Canada E-mail: helene.kuntz@queensu.ca 


\section{APPENDICES}

APPENDIX 1. Search strategy example.

\begin{tabular}{|ll|}
\hline \multicolumn{1}{|c|}{ Eligibility Criteria } & \multicolumn{1}{c|}{ Search Term } \\
Intellectual and & Intellectual impairment \\
developmental & Mental deficiency \\
disabilities & Multiple malformation syndrome \\
& Metabolic encephalopathy \\
& Congenital hypothyroidism \\
& Mental patient \\
& Autism \\
& Developmental disorder \\
& Learning disabilit\$ \\
Frailty & Frail\$ \\
\hline
\end{tabular}

